\title{
Year in Review 2015: Extracorporeal Membrane Oxygenation
}

\author{
Lakshmi Raman MD and Heidi J Dalton MD MCCM
}

\author{
Introduction \\ History \\ ECMO in Pediatric ARDS \\ Characterizing Acute Lung Injury \\ Dead-Space Ventilation and Association With Mortality \\ International Survey on Ventilation Practices on ECMO \\ Bleeding and Thrombosis on ECMO and Comparison With Pump Types \\ Early Mobilization of Patients Receiving ECMO \\ Hospital Volume of ECMO Cases and Mortality \\ Refractory Cardiac Arrest and ECMO (CHEER Trial) \\ Summary
}

\begin{abstract}
Extracorporeal membrane oxygenation (ECMO) is a modified form of cardiopulmonary bypass. Although early trials were plagued by severe bleeding and high rates of death, subsequent experience with neonates found good survival, and ECMO became an important tool in the care of critically ill infants with respiratory failure. Since the 1980s, expansion to other groups (children, patients with cardiac disease, etc) followed as experience was obtained. Today, there is a rapid growth of ECMO, especially in the adult population. To date, $>\mathbf{7 3 , 0 0 0}$ patients receiving ECMO have been reported to the international Extracorporeal Life Support Organization registry. This rapid growth in the usage of ECMO has made it possible for it to be included in the management algorithm of certain disease processes, such as ARDS, cardiopulmonary arrest, and septic shock. Significant advances in technology have made it possible to support patients on ECMO for weeks or months with success. Reduction in sedative use and experience with "awake" patients has led to ambulatory and mobile ECMO. Changes in ventilator support while on ECMO, even to the point of extubation, are also occurring. This article will review briefly some of the literature related to criteria for severity of illness before ECMO and related to ECMO care and practice. Issues relating to the use of ECMO as a resuscitative tool in cardiac arrest as well as the controversial topic of volume and outcome will also be presented. Key words: extracorporeal membrane oxygenation (ECMO); pediatric ARDS; cardiac arrest; regionalization; bleeding; thrombosis. [Respir Care 2016;61(7):986-991. @ 2016 Daedalus Enterprises]
\end{abstract}

\section{Introduction}

Extracorporeal life support, more commonly referred to as extracorporeal membrane oxygenation (ECMO), is a modified form of cardiopulmonary bypass. During ECMO, blood is removed from the venous side and pumped through an extracorporeal circuit containing an artificial lung (mem-

Dr Dalton presented a version of this paper at the Year in Review 2015 of the AARC Congress 2015, held November 7-10, 2015, in Tampa, Florida. 
brane oxygenator) in which oxygen is added and carbon dioxide is removed. Blood is then returned to the patient either through the venous side of the circulation in the case of veno venous ECMO or to the arterial side in the case of venoarterial ECMO. ${ }^{1,2}$

\section{History}

ECMO was first successfully used in 1974 in a trauma victim with ARDS. ${ }^{3}$ Subsequently, ECMO was pioneered as a technique to support term or near-term infants with severe respiratory failure. ${ }^{1,4,5}$ The efficacy of ECMO has been supported by evidence from one large randomized, controlled trial and several smaller trials. Importantly, the successful use of ECMO in infants established ECMO as a technical success, which led to its expansion in other critical care populations..$^{6-8}$ The rise of ECMO has led to an increase in the number of publications related to patient care and practice. A description of some of the literature from the recent past is shared below. Readers are encouraged to read the full text of these reports, since only a short summary is presented here.

\section{ECMO in Pediatric ARDS}

After 2 years of work, the Pediatric Acute Lung Injury Consensus Conference (PALICC), a group of experts from around the world, published their work on defining pediatric respiratory failure and recommendations for patient care in a supplement of Pediatric Critical Care Medicine. ${ }^{9}$ Eleven different topics are included, such as epidemiology, severity scoring definitions, pathophysiology, suggestions for best mechanical ventilation and noninvasive ventilation settings, and others. An excerpt from this supplement regarding ECMO is included here.

The accepted wording was confirmed as pediatric ARDS. Mechanical ventilation is the cornerstone of management in pediatric ARDS. However, mechanical ventilation is known to be associated with further lung injury through overdistention, cyclical opening and closing of alveolar units, and oxygen toxicity. Although protective lung strategies are often adopted, these protective thresholds are often exceeded to maintain adequate gas exchange. The role of ECMO in respiratory failure is also discussed. OverDr Dalton has disclosed relationships with Innovative ECMO Concepts
Inc and Maquet. Dr Raman has disclosed no conflicts of interest.

Correspondence: Heidi J Dalton MD, Inova Fairfax Hospital, 3300 Gallows Road, Falls Church, VA 22042. E-mail: Heidi.dalton@inova.org.

DOI: $10.4187 /$ respcare. 04985 all survival of pediatric respiratory failure patients with ECMO averages 56\%.

There are no clear agreed-upon criteria for the provision of ECMO support for children with pediatric ARDS. Two clinical measures often used at the bedside are oxygenation index (OI), which is a composite measure of oxygenation and ventilator support [(mean airway pressure $X$ $\left.\mathrm{F}_{\mathrm{IO}_{2}}\right) 100 / \mathrm{P}_{\mathrm{aO}}$ ] and $\mathrm{P}_{\mathrm{aO}_{2}} / \mathrm{F}_{\mathrm{IO}_{2}}{ }^{10,11} \mathrm{~A}$ multi-center review has shown that $\mathrm{OI}$ is a predictor of mortality in pediatric respiratory failure. ${ }^{10,12}$ In the randomized trial of ECMO versus conventional therapy, OI was an independent predictor of mortality. Also in stratified mortality risk, subjects receiving ECMO had significantly lower mortality compared with risk-matched pairs. Because there is a lack of clear criteria applicable to individual patients, decisions to place patients receiving ECMO support are recommended based on serial clinical trends.

Recommendations from the pediatric ARDS consensus group regarding the use of ECMO are as follows. ${ }^{13}$

- Children with severe pediatric ARDS where the cause of the respiratory failure is believed to be reversible or the child is likely to be suitable for consideration for lung transplantation. Strong agreement.

- It is not possible to apply strict criteria for the selection of children who will benefit from ECMO in pediatric ARDS. We recommend that children with severe pediatric ARDS should be considered for ECMO when lung-protective strategies result in inadequate gas exchange. Strong agreement.

- We recommend that decisions to institute ECMO should be based on a structured evaluation of case history and clinical status. Strong agreement.

- We recommend that serial evaluation of ECMO eligibility is more useful than single-point assessment. Strong agreement.

- We recommend that careful consideration of quality of life and likelihood of benefit should be assessed. Strong agreement.

\section{Characterizing Acute Lung Injury}

Yehya et al ${ }^{14}$ examined subjects in a single center and compared them with some of the findings in the PALICC reports. The timing of their study, appearing at about the same time as the PALICC supplement, brings some validation to the recommendations by the PALICC group. To characterize variables associated with mortality and ventilator-free days in acute respiratory distress, 283 children with ARDS from a single center were prospectively studied. This study found that initial OI or $\mathrm{P}_{\mathrm{aO}_{2}} / \mathrm{F}_{\mathrm{IO}_{2}}$ might poorly predict outcome. However, the same measurements after the first $24 \mathrm{~h}$ of stabilization may be more predictive of lung injury, allowing time for lung recruitment and resuscitation to take place. 
In this study, both the $\mathrm{P}_{\mathrm{aO}} / \mathrm{F}_{\mathrm{IO}_{2}}$ and $\mathrm{OI}$ at $24 \mathrm{~h}$ were predictive of mortality $(P<.001$ and $P=.002$, respectively). Similarly, the worst $\mathrm{P}_{\mathrm{aO}_{2}} / \mathrm{F}_{\mathrm{IO}_{2}}$ and worst $\mathrm{OI}$ in the first $24 \mathrm{~h}$ were also predictive of mortality $(P<.001$ and $P=.002$, respectively). The $\mathrm{P}_{\mathrm{aO}_{2}} / \mathrm{F}_{\mathrm{IO}_{2}}$ and $\mathrm{OI}$ at $24 \mathrm{~h}$ and worst $\mathrm{P}_{\mathrm{aO}_{2}} / \mathrm{F}_{\mathrm{IO}_{2}}$ and worst $\mathrm{OI}$ in the first $24 \mathrm{~h}$ were predictive of ventilator-free days from 0 to $28 \mathrm{~d}$ and ventilator-free days $\leq 14 \mathrm{~d}(P<.001)$. Increasing extrapulmonary organ failure at ARDS diagnosis correlated with increasing mortality, with the worst 24 -h values discriminating the mortality. This was also true for subjects who were immunocompromised. The impact of inhaled nitric oxide therapy on mortality was also examined in subgroup analysis. In this subgroup of subjects, the $24-\mathrm{h} \mathrm{P}_{\mathrm{aO}_{2}} / \mathrm{F}_{\mathrm{IO}_{2}}$ and OI predicted mortality, ventilator-free days, and duration of ventilation in survivors.

\section{Dead-Space Ventilation and Association With Mortality}

Another study by Yehya et al ${ }^{15}$ followed other reports on serial measures of respiratory failure and outcome in children. ${ }^{12,16}$ This report focuses on the effect of dead space and risk of mortality. ${ }^{15}$ Dead space is defined as areas of the lung that do not participate in gas exchange either due to lack of ventilation or lack of blood flow. Mechanical ventilation is associated with higher dead space. In studies of adults with ARDS, elevated dead space has been shown to be associated with mortality. ${ }^{15,17-19}$ Multiple small study cohorts in children with acute hypoxemic respiratory failure have shown an association between increased dead-space ventilation and mortality after controlling for oxygenation defect. ${ }^{17,19}$ In a large single-center study with 712 children mechanically ventilated for acute hypoxemic respiratory failure, increased dead space was associated with higher mortality. For every increase of 0.1 in dead space, the odds ratio of death was 1.39. This was true as an independent variable, although when controlled for other factors, such as OI, inotrope use, and severity of illness, it was not associated with increased mortality.

\section{International Survey on Ventilation Practices on ECMO}

One conundrum regarding the care of patients receiving ECMO is the optimal mechanical ventilator support during the ECMO course. This issue was evaluated in a survey published in 2014 among international centers for patients receiving veno venous ECMO ${ }^{20}$ Although surveys must be interpreted with caution, since actual practice may vary from survey results, it does provide new information. Other similar evaluations are also being completed, and whether results will be different should be evident as new publications appear.
The optimal ventilation strategy for mechanical ventilation when patients are receiving veno venous ECMO is unclear, and practices vary widely. To answer this question, Marhong et $\mathrm{al}^{20}$ sent out an international survey to centers registered with the international Extracorporeal Life Support Organization (ELSO). A cross-sectional survey of medical directors and ECMO program coordinators was conducted. The survey collected data on the following domains: (1) patient population and center characteristics; (2) the presence or absence of an explicit mechanical ventilation protocol; (3) mechanical ventilator settings; and (4) weaning practices. A total of 141 responses from 6 continents were included in the final analysis. The primary goal of mechanical ventilation on veno venous ECMO was lung rest (77\%). Only $27 \%$ of the centers had an explicit mechanical ventilation management protocol. The majority used a controlled mode of ventilation (62\%). PEEP levels of $>5 \mathrm{~cm} \mathrm{H}_{2} \mathrm{O}$ were used in $80 \%$ of centers, whereas $58 \%$ of centers targeted PEEP of $6-10 \mathrm{~cm} \mathrm{H}_{2} \mathrm{O}$. Thirtyone percent of the centers used ultraprotective tidal volumes $(<4 \mathrm{~mL} / \mathrm{kg})$ for ventilation on ECMO. Weaning from veno venous ECMO was achieved by reduction of sweep gas flow (43\%) and blood flow (21\%). No difference was seen in weaning strategy between centers that used a protocol and centers that did not have a protocol for ventilator management. Interestingly, $10 \%$ of the centers preferred weaning from the ventilator before removal from the ECMO circuit.

\section{Bleeding and Thrombosis on ECMO and Comparison With Pump Types}

Exposure to an ECMO circuit increases the risk of bleeding and thrombosis significantly, thereby increasing morbidity and mortality. ${ }^{21-23}$ To evaluate the bleeding and thrombotic complications, Dalton et $\mathrm{al}^{24}$ analyzed the ELSO registry data from 8 of the Collaborative Pediatric Critical Care Research Network (CPCCRN) centers for a 7-y period (2005-2011). The study outcome was to describe the bleeding and thrombotic complications and survival to hospital discharge. Bleeding complications were defined as intracranial, gastrointestinal, or pulmonary hemorrhage; cannula site bleeding; surgical site bleeding; hemolysis $(>50 \mathrm{mg} / \mathrm{dL})$, and disseminated intravascular coagulation. Thrombotic complications were divided into intracranial infarction and clots in the oxygenator, bridge, bladder, hemofilter, or other. The report was a prelude to a prospective evaluation of specific factors related to patient care, ECMO technology, and many other data elements among CPCCRN centers in a rigorous manner to obtain more specific results. Another report focused on the association of pump type used during ECMO, either roller or centrifugal, since many centers are changing modalities. ${ }^{25}$ 
Over the study period, there were 2,036 subjects receiving ECMO, of whom 263 were receiving it for congenital diaphragmatic hernia. ${ }^{24}$ Complications steadily declined for both neonatal and pediatric subjects over the study years (trend $P<.001$ ). Bleeding complications occurred in $33 \%$ of neonates and $45 \%$ of pediatric subjects other than congenital diaphragmatic hernia. The most common bleeding events included surgical site bleeding, cannula site bleeding, and intracranial hemorrhage. Bleeding and thrombotic complications were associated with decreased survival. In subjects requiring ECMO for congenital diaphragmatic hernia, bleeding and thrombotic complications occurred in 45 and $60 \%$, respectively.

In 2010-2011, there was a change in pump technology, with many centers moving from roller head pumps to centrifugal pumps. To compare the bleeding complications between the 2 pump technologies, a single-center study from the University of Michigan examined adult subjects receiving ECMO support for $\geq 5 \mathrm{~d}$ from 2002 to $2013 .{ }^{25}$ Of 95 adults, 47 were supported with the roller pump, and 48 of them received centrifugal pump support. There was no difference in the bleeding complications between the 2 pump types. However, when complications were grouped as surgical (cannulation site and surgical site) versus nonsurgical bleeding (pulmonary, gastrointestinal, and neurological), there was a higher incidence of non-surgical bleeding with the centrifugal pump. Survival to decannulation and survival to hospital discharge were both higher with roller head pumps.

\section{Early Mobilization of Patients Receiving ECMO}

Given the high morbidity of patients in the ICU with muscle weakness, decubiti, and other adverse events, coupled with new ECMO technology that makes patient movement easier and potentially safer, there is a strong effort to minimize sedation and allow patients to be awake, even walking and participating in physical therapy. ${ }^{26,27}$ In the early days, ECMO was accomplished by heavy sedation and most often by neuromuscular blockade because patients were considered very unstable. With advances in technology and longer duration of ECMO and as patients are being supported as a bridge to transplant, early mobilization has become very important. Small case series with reports of extubation and early tracheostomy with ambulation is being increasingly utilized.

Abrams et $\mathrm{al}^{28}$ conducted a retrospective review of one center's experience with early ambulation with a subset of their ECMO patients being managed by a multidisciplinary team. These 35 subjects mainly included individuals with respiratory failure either as a bridge to transplant or bridge to recovery from their respiratory illness. They used a mobilization scale of 1-8 with 1 being no mobilization or passive range of motion and 8 being full ambulation. Sub- jects were assessed for suitability by the medical team, followed by evaluation by a physical therapist/occupational therapist for level of activity. They were then prepared for the activity, and then therapy was initiated. Survival was $53 \%$ in the bridge to transplant group and $88 \%$ in the bridge to recovery group with an average ECMO time of $13.8 \pm 7.8$ and $9.1 \pm 2.6 \mathrm{~d}$ in each of the groups, respectively. There was also an increase in participation in physical therapy from the start of the program in 2009 to 2011. They did not have any patient- or circuit-related complications. ${ }^{28}$

\section{Hospital Volume of ECMO Cases and Mortality}

Providing ECMO support requires experienced skilled personnel. There has been a significant rise in the number patients being supported by ECMO and, with that, a growing number of centers, particularly for adult patients. This has raised some concerns regarding rapid diffusion of lowvolume advanced care modalities compromising efficacy and effectiveness. One fairly recent white paper of international ECMO experts recommended that ECMO centers providing adult respiratory ECMO should perform at least 20 cases annually. ${ }^{29}$ Also, some recent pediatric studies have shown that higher ECMO volume is associated with lower mortality. ${ }^{30}$

A study by Barbaro et $\mathrm{al}^{31}$ examined the international ELSO registry to evaluate the relationship between ECMO volume and mortality in 3 different age groups that included neonatal, pediatric, and adult subjects from January of 1989 to December of 2013. Volume was divided into 4 categories based on yearly patient cases reported to ELSO: $<6,6-14,15-30$, and $>30$. With the volume of cases as a continuous variable, higher ECMO volume was associated with lower in-hospital mortality in neonatal and adult subjects but not in pediatric subjects using a hierarchical regression model. When single level regression analysis was used, a difference was also noted in the pediatric age group. A subgroup analysis was done for the years 2008 2013 to account for advancement in technology and expansion in the number of adults, which showed that the volume-outcome relationship persisted for adults but not for neonatal or pediatric age groups. In a contrasting report $^{32}$, propensity core matching looking at ECMO support for children with congenital heart disease, no relationship was found between ECMO volume and mortality. However, low volume was defined as $0-30$ cases/y, medium volume was $31-50$ cases/y, and high volume was $>50$ cases/y.

\section{Refractory Cardiac Arrest and ECMO (CHEER Trial)}

Both in-hospital and out-of-hospital cardiac arrest carry significant mortality. Use of ECMO in cardiopulmonary 
resuscitation (CPR) has been proposed by the American Heart Association, and ECMO has been successfully used in pediatric and neonatal CPR with good outcomes. ${ }^{33}$ The CHEER trial (cardiac arrest, hypothermia, ECMO, early reperfusion) was a prospective pilot study of a treatment protocol from Australia to examine whether ECMO in a specified algorithm of resuscitation improved outcome. ${ }^{34}$ Patients with out-of-hospital cardiac arrest were eligible if they met the following criteria: (1) age 18-65 y; (2) cardiac arrest due to suspected cardiac etiology; (3) chest compressions commenced within 10 min by bystanders; (4) initial cardiac arrest rhythm of ventricular fibrillation; and (5) mechanical CPR machine available. Patients with in-hospital cardiac arrest were also considered if the underlying cardiac arrest was thought to be reversible and had no significant co-morbidities and underlying neurological disability. Subjects with out-of-hospital cardiac arrest were transported to the Alfred Hospital with mechanical chest compressions and given rapid infusion of $2 \mathrm{~L}$ of ice-cold saline. Subjects with in-hospital cardiac arrest also had CPR with a mechanical CPR device and were cooled. Subjects were percutaneously cannulated via the femoral artery (15 French) and vein (17 French) by 2 critical care physicians. A heparin bolus was given once cannula placement was achieved and ECMO flows were established. Cooling was continued at $33^{\circ} \mathrm{C}$ for another $24 \mathrm{~h}$, followed by slow rewarming of $0.25^{\circ} \mathrm{C}$ every hour. A distal limb perfusion catheter was inserted as soon as the subject was in the ICU. A total of 26 subjects had ECPR, with 11 out-of-hospital cardiac arrests and 15 in-hospital cardiac arrests. They had 14 survivors and 12 non-survivors. Average time from collapse to initiation of ECMO was $40 \mathrm{~min}$ in the survivors and $78 \mathrm{~min}$ in non-survivors. Higher survival was obtained in the in-hospital cardiac arrest compared with out-of-hospital cardiac arrest. All survivors were discharged home directly with full neurological recovery. The authors suggest that an overall protocolized approach to ECPR appears to be associated with good outcome.

\section{Summary}

ECMO is a rapidly advancing technology with rapid expansion of its use, particularly in adults. ECMO has claimed its role in management algorithms of a number of diseases, such as ARDS and sepsis, and CPR. Challenges of bleeding and thrombosis still exist and are ongoing. Advances in technology have made it possible for patients to be supported for long durations with the ability to ambulate while receiving ECMO. The rapid expansion of this complex critical therapy in centers with few or many patients has raised the issue of volume-outcome and suggestions for regionalization. Many new reports of ECMO and related patient care are appearing, and the brief excerpts presented here will hopefully encourage readers to seek out full publications and review results.

\section{REFERENCES}

1. Bartlett RH, Andrews AF, Toomasian JM, Haiduc NJ, Gazzaniga AB. Extracorporeal membrane oxygenation for newborn respiratory failure: forty-five cases. Surgery 1982;92(2):425-433.

2. Klein MD, Andrews AF, Wesley JR, Toomasian J, Nixon C, Roloff D, Bartlett RH. Venovenous perfusion in ECMO for newborn respiratory insufficiency: a clinical comparison with venoarterial perfusion. Ann Surg 1985;201(4):520-526.

3. Hill JD. Acute pulmonary failure: treatment with extracorporeal oxygenation. Med Instrum 1977;11(4):198-201.

4. Andrews AF, Roloff DW, Bartlett RH. Use of extracorporeal membrane oxygenators in persistent pulmonary hypertension of the newborn. Clin Perinatol 1984;11(3):729-735.

5. Andrews AF, Toomasian J, Oram A, Bartlett RH. Total respiratory support with venovenous (VV) ECMO. Trans Am Soc Artif Intern Organs 1982;28:350-353.

6. Park PK, Dalton HJ, Bartlett RH. Point: efficacy of extracorporeal membrane oxygenation in 2009 influenza A(H1N1): sufficient evidence? Chest 2010;138(4):776-778.

7. Brindley PG, Cave D, Lequier L. BEST evidence in critical care medicine: extracorporeal membrane oxygenation (ECMO) in severe adult respiratory distress syndrome. Can J Anaesth 2010;57(3):273275.

8. Aissaoui N, Luyt CE, Leprince P, Trouillet JL, Léger P, Pavie A, et al. Predictors of successful extracorporeal membrane oxygenation (ECMO) weaning after assistance for refractory cardiogenic shock. Intensive Care Med 2011;37(11):1738-1745.

9. Pediatric Acute Lung Injury Consensus Conference Group. Pediatric acute respiratory distress syndrome: consensus recommendations from the Pediatric Acute Lung Injury Consensus Conference. Pediatr Crit Care Med 2015;16(5):428-439.

10. Green TP, Timmons OD, Fackler JC, Moler FW, Thompson AE, Sweeney MF. The impact of extracorporeal membrane oxygenation on survival in pediatric patients with acute respiratory failure: Pediatric Critical Care Study Group. Crit Care Med 1996;24(2):323-329.

11. Arnold JH, Truog RD, Thompson JE, Fackler JC. High-frequency oscillatory ventilation in pediatric respiratory failure. Crit Care Med 1993;21(2):272-278.

12. Khemani RG, Thomas NJ, Venkatachalam V, Scimeme JP, Berutti $\mathrm{T}$, Schneider JB, et al. Comparison of $\mathrm{S}_{\mathrm{pO}_{2}}$ to $\mathrm{P}_{\mathrm{aO}_{2}}$ based markers of lung disease severity for children with acute lung injury. Crit Care Med 2012;40(4):1309-1316.

13. Dalton HJ, Macrae DJ, Pediatric Acute Lung Injury Consensus Conference Group. Extracorporeal support in children with pediatric acute respiratory distress syndrome: proceedings from the Pediatric Acute Lung Injury Consensus Conference. Pediatr Crit Care Med 2015;16(5 Suppl 1):S111-S117.

14. Yehya N, Servaes S, Thomas NJ. Characterizing degree of lung injury in pediatric acute respiratory distress syndrome. Crit Care Med 2015;43(5):937-946.

15. Yehya N, Bhalla AK, Thomas NJ, Khemani RG. Alveolar dead space fraction discriminates mortality in pediatric acute respiratory distress syndrome. Pediatr Crit Care Med 2016;17(2):101-109.

16. Lubrano R, Cecchetti C, Elli M, Tomasello C, Guido G, Di Nardo M, et al. Prognostic value of extravascular lung water index in critically ill children with acute respiratory failure. Intensive Care Med 2011; 37(1):124-131.

17. Nuckton TJ, Alonso JA, Kallet RH, Daniel BM, Pittet JF, Eisner MD, Matthay MA. Pulmonary dead-space fraction as a risk factor for 


\section{YEAR IN REVIEW 2015: ECMO}

death in the acute respiratory distress syndrome. $\mathrm{N}$ Engl $\mathrm{J}$ Med 2002;346(17):1281-1286.

18. Coss-Bu JA, Walding DL, David YB, Jefferson LS. Dead space ventilation in critically ill children with lung injury. Chest 2003; 123(6):2050-2056.

19. Kallet RH, Zhuo H, Liu KD, Calfee CS, Matthay MA, National Heart Lung and Blood Institute ARDS Network Investigators. The association between physiologic dead-space fraction and mortality in subjects with ARDS enrolled in a prospective multi-center clinical trial. Respir Care 2014;59(11):1611-1618.

20. Marhong JD, Telesnicki T, Munshi L, Del Sorbo L, Detsky M, Fan E. Mechanical ventilation during extracorporeal membrane oxygenation: an international survey. Ann Am Thorac Soc 2014;11(6):956961.

21. Wilson JM, Bower LK, Fackler JC, Beals DA, Bergus BO, Kevy SV. Aminocaproic acid decreases the incidence of intracranial hemorrhage and other hemorrhagic complications of ECMO. J Pediatr Surg 1993;28(4):536-540; discussion 540-531.

22. Agerstrand CL, Burkart KM, Abrams DC, Bacchetta MD, Brodie D. Blood conservation in extracorporeal membrane oxygenation for acute respiratory distress syndrome. Ann Thorac Surg 2015;99(2):590595.

23. Oliver WC. Anticoagulation and coagulation management for ECMO. Semin Cardiothorac Vasc Anesth 2009;13(3):154-175.

24. Dalton HJ, Garcia-Filion P, Holubkov R, Moler FW, Shanley T, Heidemann $\mathrm{S}$, et al. Association of bleeding and thrombosis with outcome in extracorporeal life support. Pediatr Crit Care Med 2015; 16(2):167-174.

25. Halaweish I, Cole A, Cooley E, Lynch WR, Haft JW. Roller and centrifugal pumps: a retrospective comparison of bleeding complications in extracorporeal membrane oxygenation. ASAIO J 2015; 61(5):496-501.

26. Azoulay E, Citerio G, Bakker J, Bassetti M, Benoit D, Cecconi M, et al. Year in review in Intensive Care Medicine 2013: II. Sedation, invasive and noninvasive ventilation, airways, ARDS, ECMO, family satisfaction, end-of-life care, organ donation, informed consent, safety, hematological issues in critically ill patients. Intensive Care Med 2014;40(3):305-319.

27. Abrams DC, Brenner K, Burkart KM, Agerstrand CL, Thomashow BM, Bacchetta M, Brodie D. Pilot study of extracorporeal carbon dioxide removal to facilitate extubation and ambulation in exacerbations of chronic obstructive pulmonary disease. Ann Am Thorac Soc 2013;10(4):307-314.

28. Abrams D, Javidfar J, Farrand E, Mongero LB, Agerstrand CL, Ryan $\mathrm{P}$, et al. Early mobilization of patients receiving extracorporeal membrane oxygenation: a retrospective cohort study. Crit Care 2014; 18(1):R38

29. Combes A, Brodie D, Bartlett R, Brochard L, Brower R, Conrad S, et al. Position paper for the organization of extracorporeal membrane oxygenation programs for acute respiratory failure in adult patients. Am J Respir Crit Care Med 2014;190(5):488-496.

30. Freeman CL, Bennett TD, Casper TC, Larsen GY, Hubbard A, Wilkes J, Bratton SL. Pediatric and neonatal extracorporeal membrane oxygenation: does center volume impact mortality?. Crit Care Med 2014;42(3):512-519.

31. Barbaro RP, Odetola FO, Kidwell KM, Paden ML, Bartlett RH, Davis MM, Annich GM. Association of hospital-level volume of extracorporeal membrane oxygenation cases and mortality: analysis of the extracorporeal life support organization registry. Am J Respir Crit Care Med 2015;191(8):894-901.

32. Gupta P, Rettiganti M. Association between extracorporeal membrane oxygenation center volume and mortality among children with heart disease: propensity and risk modeling. Pediatr Crit Care Med 2015;16(9):868-874.

33. Goldberger ZD, Chan PS, Berg RA, Kronick SL, Cooke CR, Lu M, et al. Duration of resuscitation efforts and survival after in-hospital cardiac arrest: an observational study. Lancet 2012;380(9852):14731481.

34. Stub D, Bernard S, Pellegrino V, Smith K, Walker T, Sheldrake J, et al. Refractory cardiac arrest treated with mechanical CPR, hypothermia, ECMO and early reperfusion (the CHEER trial). Resuscitation 2015;86:88-94. 